\title{
Frequency-Swept Microwave Imaging using Multi-Source Illumination
}

\author{
Chao-Hsiung Tseng and Tah-Hsiung Chu \\ Graduate Institute of Communication Engineering \\ National Taiwan University, Taipei, Taiwan \\ Tel:886-2-23635251 ext. 541, Fax: 886-2-23638247 \\ E-mail:thc@ew.ee.ntu.edu.tw
}

\begin{abstract}
In this paper, the principle and experimental results of bistatic frequency-swept microwave imaging of continuous shape and discrete line conducting objects using simultaneous multi-source illumination are presented. Images of scattering objects reconstructed from the experimental data measured in the frequency range $7.5 \mathrm{GHz}-12.5 \mathrm{GHz}$ are shown in good agreement with the scattering object geometries.
\end{abstract}

\section{I . Introduction}

Microwave imaging has practical applications in the areas of imaging radar, remote sensing, and nondestructive evaluation. In order to obtain higher resolution and wider viewing angle of the object microwave images, acquiring enough scattering object information at different viewing and illumination angles is essential. There are different methods proposed to increase the scattering information, for instance, frequency diversity technique [1], multiplexing technique [2] and unrelated illumination method [3]. In this paper, the simultaneous multi-source illumination arrangement is exploited with the use of frequency-swept technique to enlarge the illuminated area over the scattering object surface. The scattering information is then recorded by a backward linear receiving array.

\section{Principle}

The scattering geometry is shown in Fig. 1. The scattering object is illuminated by multiple normally incident TM-polarized plane waves simultaneously. The scattered field recorded by the linear receiving array at $z=-d$ can be represented as

$$
U^{s}\left(x, z=-d, k_{0}\right)=\kappa \sum_{i=1}^{n} E_{i} \iint O_{i}\left(\bar{r}^{\prime}\right) e^{-j k_{0} \hat{k}_{i}, \vec{r}^{\prime}} G\left(\left|\bar{r}-\bar{r}^{\prime}\right|\right) d^{2} \bar{r}^{\prime}
$$

where $\kappa$ is $-j k_{0}$ for continuous scattering object and $K$ is 1 for discrete scattering object. $E_{i}$ is the amplitude of the $i$-th illuminating plane wave. $O_{i}\left(\bar{r}^{\prime}\right)$ is the partial microwave image contributed from the $i$-th source illumination. $G(|\vec{r}-\bar{r}|)$ is the Green's function in free space.

By using the plane wave expansion of the Green's function, the Fourier transformation 
of (1) can be expressed as

$$
\tilde{U}^{s}\left(k_{x}, z=-d, k_{0}\right)=\frac{-j \kappa}{2 \gamma} e^{-j \gamma^{d}} \sum_{i=1}^{n} E_{i} \tilde{O}_{i}\left(k_{x},-\gamma,-k_{0}\right) .
$$

where the Fourier transformation of $U^{s}\left(x, z=-d, k_{0}\right)$ and $O_{i}(\bar{r})$ are defined as

$$
\begin{gathered}
\tilde{U}^{s}\left(k_{x}, z=-d, k_{0}\right)=\int U^{s}\left(x, z=-d, k_{0}\right) e^{j k_{x} x} d x, \\
\tilde{O}_{i}(\bar{k})=\iint O_{i}(\bar{r}) e^{j \bar{k} \cdot \bar{r}} d^{2} \bar{r} .
\end{gathered}
$$

In order to properly extract each partial microwave image contributed from the corresponding source illumination, a set of $n$ arrangements of (2) with different illuminating amplitude $E_{i}$ are written as

$$
\left[\begin{array}{c}
\tilde{U}_{1}^{s}\left(k_{x}, z=-d, k_{0}\right) \\
\tilde{U}_{2}^{s}\left(k_{x}, z=-d, k_{0}\right) \\
\vdots \\
\tilde{U}_{n}^{s}\left(k_{x}, z=-d, k_{0}\right)
\end{array}\right]=\frac{j 2 \gamma}{\kappa} e^{j \gamma d}\left[\begin{array}{cccc}
E_{11} & E_{12} & \cdots & E_{1 n} \\
E_{21} & E_{22} & \cdots & E_{2 n} \\
\vdots & \vdots & \ddots & \vdots \\
E_{n 1} & E_{n 2} & \cdots & E_{n n}
\end{array}\right]\left[\begin{array}{c}
\tilde{O}_{1}\left(k_{x},-\gamma,-k_{0}\right) \\
\tilde{O}_{2}\left(k_{x},-\gamma,-k_{0}\right) \\
\vdots \\
\tilde{O}_{n}\left(k_{x},-\gamma,-k_{0}\right)
\end{array}\right] .
$$

Equation (5) shows that each partial microwave image data can be extracted from the scattering data in the Fourier-space as the matrix $\left[E_{i j}\right]$ is non-singular. Therefore, by combining partial image data in the Fourier space, microwave image with a higher resolution and wider viewing angle can be achieved by taking a two-dimensional inverse Fourier transformation.

\section{Measurement system and Results}

Figure 2 shows the developed experimental microwave imaging system. It has two horn antennas at about $\pm 30^{\circ}$ for simultaneously transmitting swept-frequency microwave signals with power levels controlled by two attenuators. An open-ended WR-90 waveguide is used as a receiving probe, located on a $158 \mathrm{~cm}$ long linear scanner at about $z=-100 \mathrm{~cm}$ for collecting the object scattered field. A Hughes $8010 \mathrm{H}$ TWTA and an Avantek AWT-18676 LNA are connected with an HP8510C network analyzer to provide proper signal amplification. A personal computer is linked to the measurement system for receiving probe movement, instrument control, system calibration, and data recording. In the measurement, the frequency is stepped from 7.5 to $12.5 \mathrm{GHz}$ for 51 frequency points and a total of 128 positions are scanned along the linear scanner.

Figure 3 shows the measured and simulation Fourier-space data and their reconstructed images for a metallic cylinder with $15 \mathrm{~cm}$ radius. The experimental results are shown in good agreement with the simulation results. The reconstructed image shows an about $60^{\circ}$ circular ring image corresponding to the shape of the metallic cylinder. In addition, measured results of the Fourier-space data and reconstructed image of four thin cylinders are illustrated in Figure 4. The reconstructed image is shown in good agreement with the distribution geometry 
of thin cylinders.

\section{Conclusions}

In this paper, the bistatic frequency-swept microwave imaging of perfectly conducting objects in a simultaneous multi-source illumination arrangement has been experimentally demonstrated. The experimental results shows that using multi-source illumination can provide a cost-effective approach for a wider imaging viewing angle and higher resolution of the scattering object.

\section{Reference}

[1] N. H. Farhat, "Microwave diversity imaging and automated target identification based on models of neural networks," Proc. IEEE, vol.77, no.5, pp.670-680, 1989.

[2] F. C. Chen and W. C. Chew, "Code-division multiplexing and frequency-division multiplexing for nonlinear inverse scattering," IEEE AP-S 1999, vol.3, pp.2128 -2131, 1999.

[3] Wang Weiyan and Zhang Shourong, "Unrelated illumination method for electromagnetic inverse scattering of inhomogeneous lossy dielectric bodies," IEEE Trans. Antenna Propagat., vol.40, pp.1292-1296, Nov. 1992.

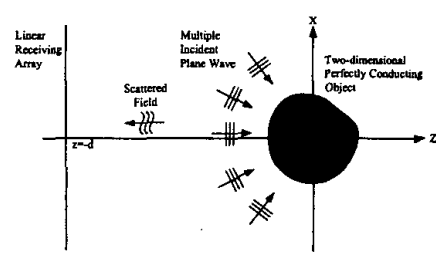

Fig.1. Two-dimensional bistatic scattering geometry using multi-source illumination.

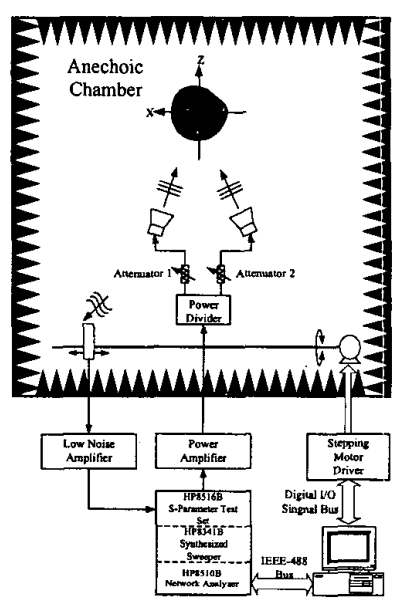

Fig. 2. An automated wide-band bistatic microwave imaging scattering measurement system using multi-source illumination. 


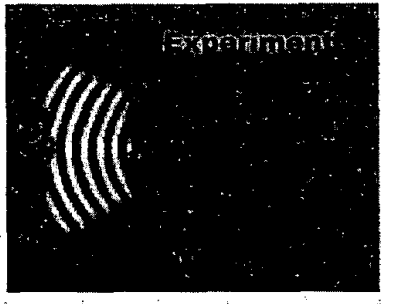

is:
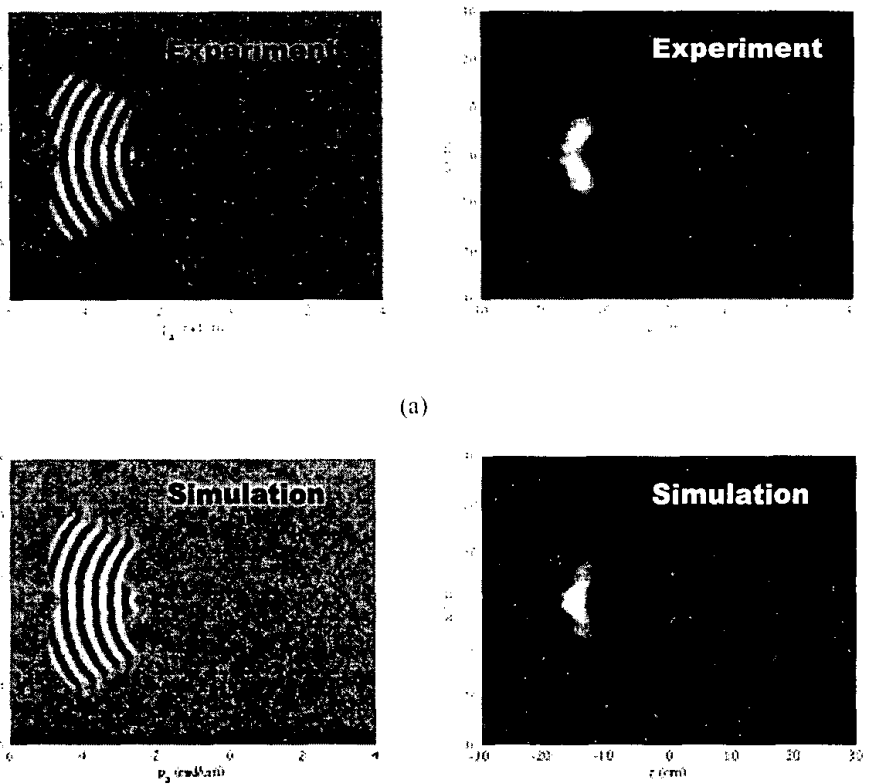

(b)

Fig. 3. (a) Measured and (b) simulation results of the Fourier space data and reconstructed images of a metallic cylinder with $15 \mathrm{~cm}$ radius.

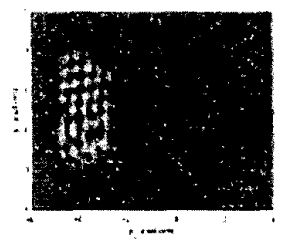

(a)

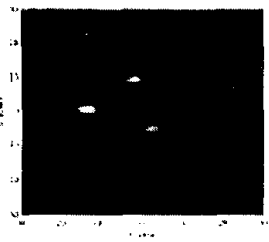

(b)

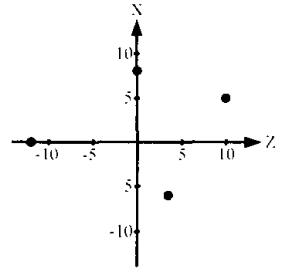

(c)

Fig. 4. Measured results of (a) Fourier-space data and (b) reconstructed image of four thin cylinders with (c) geometries at $(-12,0) \mathrm{cm},(0,8) \mathrm{cm},(3.5,-6) \mathrm{cm}$, and $(10,5) \mathrm{cm}$ respectively. 